\title{
Heparin-binding defective lipoprotein lipase is unstable and causes abnormalities in lipid delivery to tissues
}

\author{
E. Peer Lutz, ${ }^{1}$ Martin Merkel,,${ }^{2,3}$ Yuko Kako, ${ }^{1}$ Kristan Melford, ${ }^{4}$ Herbert Radner, ${ }^{5}$ \\ Jan L. Breslow, ${ }^{3}$ André Bensadoun, ${ }^{4}$ and Ira J. Goldberg ${ }^{1}$ \\ ${ }^{1}$ Department of Medicine, Columbia University, New York, New York, USA \\ ${ }^{2}$ Department of Medicine, University of Hamburg, Hamburg, Germany \\ ${ }^{3}$ Laboratory for Biochemical Genetics and Metabolism, Rockefeller University, New York, New York, USA \\ ${ }^{4}$ Division of Nutritional Sciences, Cornell University, Ithaca, New York, USA \\ ${ }^{5}$ Department of Neuropathology, University of Bonn, Medical Center, Bonn, Germany
}

Address correspondence to: Ira J. Goldberg, Department of Medicine, Columbia University, 630 West 168th Street, New York, New York 10032, USA. Phone: (212) 305-3678; Fax: (212) 305-5384; E-mail: ijg3@columbia.edu.

Received for publication November 13, 2000, and accepted in revised form March 24, 2001.

Lipoprotein lipase (LPL) binding to heparan sulfate proteoglycans (HSPGs) is hypothesized to stabilize the enzyme, localize LpL in specific capillary beds, and route lipoprotein lipids to the underlying tissues. To test these hypotheses in vivo, we created mice expressing a human LpL minigene $\left(\mathrm{hLpL}^{\mathrm{HBM}}\right.$ ) carrying a mutated heparin-binding site. Three basic amino acids in the carboxyl terminal region of $\mathrm{LPL}$ were mutated, yielding an active enzyme with reduced heparin binding. Mice expressing $\mathrm{hLPL}^{\mathrm{HBM}}$ accumulated inactive human $\mathrm{LpL}(\mathrm{hLpL})$ protein in preheparin blood. $\mathrm{hLpL} \mathrm{L}^{\mathrm{HBM}}$ rapidly lost activity during a $37^{\circ} \mathrm{C}$ incubation, confirming a requirement for heparin binding to stabilize LpL. Nevertheless, expression of $h \mathrm{LpL}^{\mathrm{HBM}}$ prevented the neonatal demise of $\mathrm{LpL}$ knockout mice. On the LpL-deficient background $\mathrm{hLPL}^{\mathrm{HBM}}$ expression led to defective targeting of lipids to tissues. Compared with mice expressing native $\mathrm{hLpL}$ in the muscle, $\mathrm{hLpL} \mathrm{LBM}^{\mathrm{HBM}}$ transgenic mice had increased postprandial FFAs, decreased lipid uptake in muscle tissue, and increased lipid uptake in kidneys. Thus, heparin association is required for LpL stability and normal physiologic functions. These experiments confirm in vivo that association with HSPGs can provide a means to maintain proteins in their stable conformations and to anchor them at sites where their activity is required.

J. Clin. Invest. 107:1183-1192 (2001).

\section{Introduction}

Lipoprotein lipase (LpL) hydrolyzes circulating triglyceride (TG) into FFAs while it is bound to the luminal surface of endothelial cells (1). Several lines of evidence suggest that this interaction is highly dependent on LpL binding to endothelial cell surface heparan sulfate proteoglycans (HSPGs): (a) LpL is a high affinity heparin-binding protein (2); (b) LpL is released into the bloodstream of humans and animals by intravenous injection of heparin; (c) heparinase treatment of endothelial cells markedly reduces LpL binding to endothelial cells (3); and (d) high affinity LpL-binding proteoglycans (4) and heparan-sulfate oligosaccharides $(5,6)$ have been isolated from cultured endothelial cells. Heparin binding is important for biological actions of a number of proteins including enzymes, growth factors, cytokines, and coagulation proteins. In some situations, association with HSPGs appears to concentrate proteins near signaling and endocytosis receptors (7). This, for example, allows maximum function of bFGF (8). Heparin binding had been postulated to require consensus sequences containing clusters of basic amino acids containing the sequences XBBXBX and XBBBXXBX (9). However, more recent reviews of heparin-binding proteins have concluded that this simplistic approach to defining heparin-binding regions without consideration of the tertiary and quaternary structure of proteins has often been incorrect $(10,11)$. Aside from its importance in lipid/lipoprotein metabolism, LpL is a model protein for studying physiologic implications and biochemistry of heparin binding and protein dimerization. LpL association with HSPGs is postulated, from in vitro data, to affect LpL metabolism at three separate steps. LpL binding to HSPGs on the luminal side of endothelial cells concentrates LpL in sites of enzyme synthesis like the adipose and muscles. This is hypothesized to promote local uptake of LpL-generated fatty acids into those tissues. A second role of LpL binding to HSPGs may be to stabilize the enzyme on its endothelial-binding site. In vitro data have demonstrated that $\mathrm{LPL}$ is rapidly inactivated at $37^{\circ} \mathrm{C}$, and this loss of activity is partially prevented by inclusion of heparin in the incubation. Finally, heparin binding may be required for efficient removal of LpL from the bloodstream. Support for this role of heparin binding comes from experiments showing that heparin injection into animals delays clearance of LpL by the liver (12).

LpL has several clusters of basic amino acids that were thought to represent heparin-binding domains. When a postulated heparin-binding region in the amino terminal half of LpL was mutated, LpL-heparin interaction 
was decreased but not eliminated (13-15). This suggested that a second site was involved and probably represented the major heparin-binding region. By creating chimeric molecules using regions of $\mathrm{LpL}$ and hepatic lipase, it was shown that high affinity heparin binding requires the carboxyl terminal region of $\operatorname{LpL}(16,17)$; this region does not contain a consensus heparin-binding sequence. While some in vitro experiments suggested that the carboxyl-terminal region of LpL contains a major heparin-binding region (18), other experiments using bacterially expressed LPL fragments failed to confirm this (19). Thus, although heparin binding is a characteristic of LpL, the region(s) of the molecule required for HSPG binding has not been defined in vivo and the in vitro experiments are conflicting.

To determine whether the carboxyl terminal region of human $\mathrm{LpL}$ (hLpL) is important for HSPG binding in vivo and to assess the biological effects of having heparin-binding mutated LpL, we produced mutations in the carboxyl terminal region of hLpL corresponding to mutations in avian LpL cDNA that lead to a significant loss in heparin binding (18) and studied their physiologic consequences in a transgenic mouse model.

\section{Methods}

Construction of buman heparin-binding mutated LPL ( $b L P L$ $H B M)$. A PCR-based site-specific mutagenesis of the muscle creatine kinase-hLpL (MCK-hLpL) minigene (20) was performed using a kit (Stratagene, La Jolla, California, USA) and the following primers: Primer 1: GCT TCG CCA TTC AGA ACA TCA ATG TAA ATG CAG GAG AGA CTC AG, primer 2: CTG AGT CTC TCC TGC ATT TAC ATT GAT GTT CTG AAT GGC GAA GC. This procedure converted the coding regions for amino acids 403,405 , and 407 of the hLpL cDNA from $\mathrm{Arg}^{403}$ to $\mathrm{Asn}^{403}, \mathrm{Arg}^{405}$ to $\mathrm{Asn}^{405}$, and $\mathrm{Lys}^{407}$ to $\mathrm{Asn}^{407}$.

The mutated hLpL ${ }^{\mathrm{HBM}} \mathrm{cDNA}$ was cloned in a pcDNA3.1 vector in which expression is driven by the cytomegalovirus promoter. This plasmid was stably transfected in Chinese hamster ovary cells (CHO-K1) as described (18). The nonmutated hLpL expressing $\mathrm{CHO}$ cells were a generous gift by N. Yamada (Institute of Clinical Medicine, University of Tsukuba, Tsukuba, Japan) (21). Cells were maintained as described previously (18). For LpL activity assays confluent cells were incubated with serum-free medium (DMEM 2\% BSA) for 2 hours. The media were collected and immediately frozen at $-70^{\circ} \mathrm{C}$.

Generation of $h L p L^{H B M}$ transgenic mice. The purified MCK-hLpL ${ }^{\text {HBM }}$ minigene was microinjected into the pronuclei of fertilized mouse eggs taken from superovulated $(\mathrm{C} 57 \mathrm{BL} / 6 \mathrm{~J} \times \mathrm{CBA} / \mathrm{J})$ F1 females as described (22). Founder animals were crossed with C57BL/6 mice (The Jackson Laboratory, Bar Harbor, Maine, USA). hLpL ${ }^{H B M}$ transgenic mice were then bred with heterozygote LpL knockout mice that had been bred more than five generations onto C57BL/6 (LpL1, ref. 23). Pups, which carried the hLpL ${ }^{\text {HBM }}$ transgene as well as the disrupted mouse LpL allele, were crossed again with
LPL1 mice. This cross resulted in the generation of $12.5 \%$ of pups that were homozygous for the $\mathrm{LpL}$ knockout allele and heterozygous for the hLpL ${ }^{\mathrm{HBM}}$ transgene. hLpL transgenic mice were bred in the same manner. From this breeding scheme, each line of mice had $\geq 93.75 \%$ C57BL/ 6 background.

Genotyping of transgenic mice. Tail-tip DNA was screened by PCR. The genotype at the mouse LPL locus was analyzed by the 3 primer PCR as described. The $h L L^{\mathrm{HBM}}$ and the hLpL transgenes were detected by utilizing the hLPL PCR (24).

LPL mass and activity measurements. To obtain postheparin plasma, fasted mice were bled 5 minutes after a tail vein injection of $100 \mathrm{U}$ heparin/kg body weight (ElkinsSinns Inc., Cherry Hill, New Jersey, USA). Human and murine LPL protein was measured by ELISA as described previously by Peterson et al. (25). LpL activity was measured by the method described by Hocquette et al. (26). To distinguish lipolysis mediated by hLpL, mouse LpL, and hepatic lipase, the mouse plasma samples were assayed in the presence of an mAb against hLpL (27) and under high salt conditions ( $1 \mathrm{M} \mathrm{NaCl}$ final concentration).

Heparin-Sepharose chromatography. Affinity chromatography was performed using an FPLC-System (Amersham Pharmacia Biotech, Piscataway, New Jersey, USA) with a $1 \mathrm{ml}$ Hi-Trap heparin-Sepharose column (Amersham Pharmacia Biotech) at $4^{\circ} \mathrm{C}$ essentially as described (18). Murine and hLpL concentrations in the fractions were determined by ELISA as described.

Gel filtration of postheparin plasma. Five hundred microliters pooled postheparin plasma from male mice $(n=4$ of each genotype, hLpL ${ }^{\mathrm{HBM}} / \mathrm{LpL} 0$ and $\mathrm{hLpL} / \mathrm{LpLO}$ ) was chromatographed by FPLC using two Superose 6 columns in series (Amersham Pharmacia Biotech) as described (28). Twenty-five fractions of $1.08 \mathrm{ml}$ were collected and assayed for LpL activity, hLpL mass, and cholesterol as described above.

Plasma lipid and lipoprotein analysis. The mice were fed a chow diet ( $4.5 \% \mathrm{fat}$, wt $/ \mathrm{wt})$. Unless stated differently all plasma samples were collected after 8 hours of daytime fasting. Plasma TG and cholesterol were determined with kits (Sigma-Aldrich, St. Louis, Missouri, USA) in duplicates. For lipoprotein analysis individual mouse plasma samples $(60 \mu \mathrm{l})$ were centrifuged twice in a Beckman TLA 100 rotor (Beckman Instruments Inc., Palo Alto, California, USA) as described (29). FFAs were determined after overnight fasting and in the postprandial state after gavage of $100 \mu \mathrm{l}$ corn oil (1 hour, 2 hours, and 3 hours postprandial). FFAs were measured in duplicates utilizing a commercial kit (NEFA C; Wako Pure Chemicals Industries Ltd., Osaka, Japan).

Northern blot. RNA was prepared from 8-week-old $h L L^{H B M} / L p L 0$ and hLpL/LpLO female mice using a kit (TRIzol Reagent; Life Technologies Inc., Rockville, Maryland, USA). The blot was hybridized with a probe spanning the $\sim 500 \mathrm{bp}$ RsRII/HindIII fragment from the hLpL minigene and then autoradiographed. A second hybridization with an oligonucleotide complementary to $18 \mathrm{~S}$ rRNA (30) was performed. 
LPL stability. Serum-free cell culture media from $\mathrm{CHO}$ cells expressing either nonmutated $\mathrm{hLpL}$ or $\mathrm{hLpL}^{\mathrm{HBM}}$ were harvested as described before. To one batch of media, $5 \mathrm{U} / \mathrm{ml}$ heparin (Elkins-Sinns) was added. Eighty microliters of the media was assayed for activity just before incubation at $37^{\circ} \mathrm{C}$ in a water bath and then after 15 minutes, 30 minutes, and 60 minutes as described above. In another experiment, two sets of three wells each on a 6-well plate of hLpL and hLpL ${ }^{\mathrm{HBM}}$ expressing $\mathrm{CHO}$ cells were used. The first set of both cell types was incubated for 4 hours with DMEM $2 \%$ BSA. The media were collected and frozen at $-70^{\circ} \mathrm{C}$. Thereafter, the cells were washed twice with ice cold PBS and then incubated with DMEM $2 \%$ BSA $+20 \mathrm{U} / \mathrm{ml}$ heparin for $30 \mathrm{~min}-$ utes at $4^{\circ} \mathrm{C}$. The media were collected and frozen. The second set of cells was only incubated with the heparin containing media as above. LpL activity was assessed in triplicates using $80 \mu \mathrm{l}$ aliquots of media. LpL stability was also assessed in postheparin plasma from $\mathrm{hLpL} / \mathrm{LpL0}$ and $\mathrm{hLpL} \mathrm{HBM}^{\mathrm{HBM}} \mathrm{LpL0}$ mice. Five microliters of 1:5 in water-diluted postheparin plasma was assayed for activity just before incubation at $37^{\circ} \mathrm{C}$ and after 30 minutes, 60 minutes, and 90 minutes.

LpL in skeletal muscle and kidneys. The mice were perfused with $20 \mathrm{ml}$ PBS prior to removal of tissues. Quadriceps, muscles, and kidneys (100 mg wet weight) were obtained from 4- to 6-month-old mice expressing only hLpL $\mathrm{LMB}^{\mathrm{HMB}}$ or hLpL. The muscles were then minced and incubated at $4{ }^{\circ} \mathrm{C}$ with DMEM $2 \% \mathrm{BSA}$ on a rocker. After 2 hours the media were collected and the tissues were washed with PBS and then homogenized as described by Hocquette et al. (26). The kidney tissues were directly homogenized (26) after removal. LpL mass in the media and tissues was measured as above.

Turnover of rat chylomicrons, Intralipid, and palmitate. Rat chylomicrons were collected and purified from the mesenteric lymph of male Wistar rats exactly as described (31) with the exception that the animals were fed $500 \mu \mathrm{Ci}$ of $\left({ }^{3} \mathrm{H}\right)$ triolein (specific activity 21 $\mathrm{Ci} / \mathrm{mmol}$, Amersham Pharmacia Biotech) and $10 \mu \mathrm{Ci}$ of $\left({ }^{14} \mathrm{C}\right)$ cholesterol (specific activity $1 \mathrm{mCi} / \mathrm{ml}$, Amersham Pharmacia Biotech) dissolved in $500 \mu \mathrm{l}$ corn oil to obtain TG and cholesterol-labeled chylomicrons. Intralipid (Kabi Pharmacia Inc., Clayton, North Carolina, USA) emulsion was labeled with $40 \mu \mathrm{Ci}$ $\left({ }^{3} \mathrm{H}\right)$ cholesteryl oleyl ether and $10 \mu \mathrm{Ci}\left({ }^{14} \mathrm{C}\right)$ triolein (Amersham Pharmacia Biotech) exactly as described by van Bennekum et al. (32). One hundred seventy microliters of the chylomicron emulsion containing $1.1 \times 10^{6}{ }^{3} \mathrm{H}$ decays per minute $(\mathrm{dpm})$ and $2 \times 10^{4}{ }^{14} \mathrm{C}$ $\mathrm{dpm}$ were injected into hLpL ${ }^{\mathrm{HBM}} / \mathrm{LpLO}(n=5)$ and hLpL/LpLO $(n=5)$ male mice at the age of 10-12 weeks via tail vein. The Intralipid emulsion was diluted in sterile saline prior to intravenous injection to $10^{6} \mathrm{dpm}\left({ }^{3} \mathrm{H}\right)$ cholesteryl oleyl ether and $3 \times 10^{5} \mathrm{dpm}$ $\left({ }^{14} \mathrm{C}\right)$ triolein in a volume of $100 \mu \mathrm{l}$. Intralipid metabolism was studied in four hLPL $\mathrm{HBM}^{\mathrm{HBM}} \mathrm{LPL}$ and four hLpL/LpLO female mice at the age of 14-16 weeks. Eighty microliters of blood was withdrawn from retroorbital plexus 2 and 4 minutes after injection. Ten minutes after injection mice were bled by cardiac puncture and then perfused with $10 \mathrm{ml}$ PBS. One hundred microliters of the plasma from the 10minute time point of the chylomicron turnover study was lipid extracted and TLC was performed using standard techniques. After perfusion the tissues were removed, homogenized, total lipids were extracted, and were counted as described (33). Fatty acid turnover utilized $\left({ }^{3} \mathrm{H}\right)$ palmitate in ethanol (NEN Life Science Inc., Boston, Massachusetts, USA; specific activity $36.3 \mathrm{Ci} / \mathrm{mmol}$ ) complexed to fatty acid free BSA (Sigma-Aldrich) as described (34). $2.2 \times 10^{6} \mathrm{dpm}$ palmitate were injected via tail vein into four hLpLHBM/LpLO and four hLpL/LpL0 male 5- to 6-monthold mice. The mice were bled from retro-orbital plexus at 15 seconds, 1 minute, 2 minutes, and 3 minutes after injection, and the plasma decay was determined.

Histological analysis. Quadriceps, soleus, and psoas muscles were dissected from 8-month-old female $h \mathrm{LL}^{\mathrm{HBM}} / \mathrm{LpL} 2$ and wild-type littermates and fixed in formaldehyde. Histological analyses including electron microscopy were performed as described (33).

\section{Results}

Generation of transgenic mice. Transgenic mice were generated that express the hLpL ${ }^{\mathrm{HBM}}$ minigene in the muscle. The expression of $\mathrm{hLpL}^{\mathrm{HBM}}$ was confirmed by measuring hLpL activity and mass in plasma. As shown in Figure 1a, $\mathrm{hLpL}$ protein was found in postheparin plasma from these mice. No hLpL protein was detected in the plasma of wild-type littermates. Surprisingly, large amounts of $\mathrm{hLpL}^{\mathrm{HBM}}$ were also found in preheparin plasma. In more than $20 \mathrm{hLpL}^{\mathrm{HBM}}$-expressing mice, plasma preheparin LpL was greater than $1.2 \mu \mathrm{g} / \mathrm{ml}$. Thus, hLpL ${ }^{\mathrm{HBM}}$ differed

\section{Figure 1}

hLpL mass and activity of hLpL ${ }^{H B M}$ transgenic mice. (a) hLpL mass in pre- and postheparin plasma of $h L p L^{H B M}$ and $h L p L$ transgenic mice. Preheparin plasma was collected immediately before intravenous injection of $100 \mathrm{U}$ heparin $/ \mathrm{kg}$. Five minutes later postheparin plasma was obtained. hLpL mass was determined by ELISA as described in Methods. (b) $\mathrm{hLpL} \mathrm{HBM}^{\mathrm{HBM}}$ and murine $\mathrm{LpL}(\mathrm{mLpL})$ activity in postheparin plasma of $h \mathrm{LpL}^{\mathrm{HBM}}$ transgenic mice and their wild-type littermates. A monoclonal antibody against $h L p L(28)$ was utilized to distinguish between $h L p L$ HBM and $m L p L$ activity. a

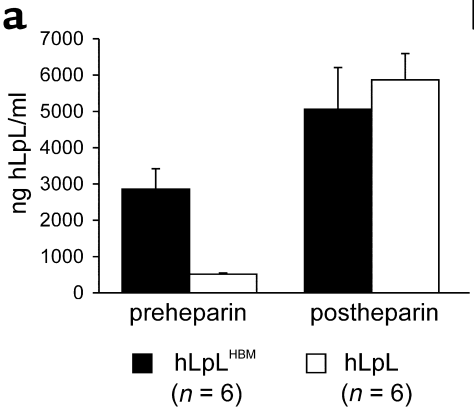

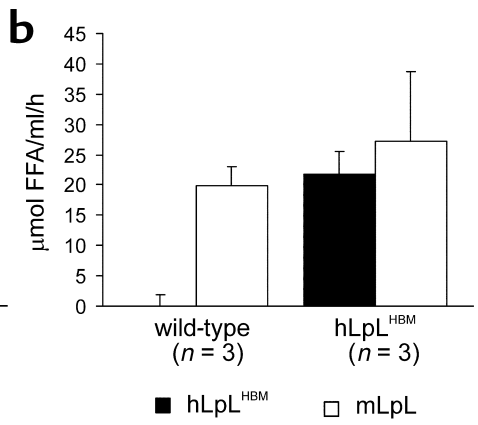




\section{Figure 2}

Heparin-affinity chromatography and gel filtration of mouse plasma. (a) Preheparin plasma from $h \mathrm{LPL}^{\mathrm{HBM}}$ transgenic mice was collected and applied to a heparin-affinity gel. LpL was eluted with a $\mathrm{NaCl}$ gradient and hLpL was measured by ELISA. (b) Postheparin plasma from hLpL ${ }^{H B M}$ transgenic mice was chromatographed on a heparinaffinity gel. Human and murine LPL masses were determined by ELISA. The amounts of $L p L$ are given as a percentage of eluted LpL per fraction. (c) Pooled postheparin plasma from hLpL ${ }^{H B M} / \mathrm{LpLO}$ mice was gel filtered on an FPLC system with two Superose 6 columns in series. LPL activity and cholesterol were determined in each fraction. (d) Pooled postheparin plasma from $\mathrm{hLpL/LpL0} \mathrm{mice} \mathrm{was} \mathrm{gel} \mathrm{filtered} \mathrm{and} \mathrm{ana-}$ lyzed as described in $\mathbf{c}$.
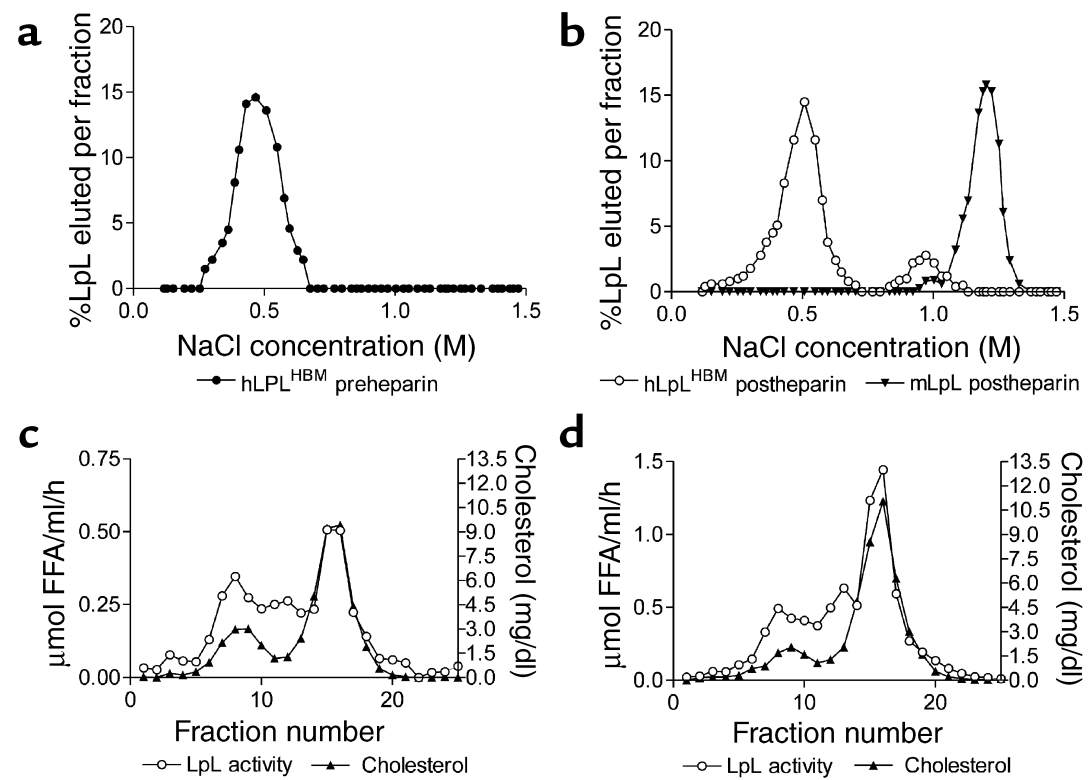

in its metabolism such that the protein was defective in its ability to remain bound to the endothelium or in its clearance from the bloodstream.

hLpL activity in postheparin plasma of hLpL ${ }^{H B M}$ transgenic mice was $21.7 \pm 3.8 \mu \mathrm{mol} \mathrm{FFA} / \mathrm{ml} / \mathrm{h}$, an amount that was similar to endogenous mouse $\mathrm{LPL}$ in these animals. Wild-type mouse postheparin plasma contained less than $0.1 \pm 1.9 \mu \mathrm{mol} \mathrm{FFA} / \mathrm{ml} / \mathrm{h}$ of hLpL activity (Figure 1b). Despite the large amount of protein, no hLpL activity was detected in the preheparin plasma of hLpL $\mathrm{HBM}_{\text {-expressing mice. }}$

Heparin-affinity chromatography of pre- and postheparin plasma from $h L p L^{H B M}$ transgenic mice. The preheparin plasma hLpL ${ }^{\mathrm{HBM}}$ protein eluted at $\sim 0.4 \mathrm{M} \mathrm{NaCl}$, Figure 2a. A second peak of $\mathrm{hLpL}^{\mathrm{HBM}}$ protein eluted at $\sim 0.9 \mathrm{M}$ $\mathrm{NaCl}$ when postheparin plasma was chromatographed (Figure 2b). Murine LpL in the postheparin plasma eluted at $\sim 1.2 \mathrm{M} \mathrm{NaCl}$. At $\sim 0.9 \mathrm{M}$ there was a small region where the hLpL ${ }^{\mathrm{HBM}}$ elution overlapped a shoulder of the murine LpL elution; this may represent heterodimers of mouse LpL and hLpL ${ }^{\mathrm{HBM}}$. These data demonstrate that plasma from mice expressing hLpL ${ }^{\mathrm{HBM}}$ contains a low affinity inactive protein in preheparin blood. Postheparin plasma appears to contain hLpL monomers and dimers, both of which are defective in heparin binding.

Breeding of $h L P L^{H B M}$ onto the $L P L O$ background. We next tested whether the hLpL ${ }^{\mathrm{HBM}}$ transgene would prevent the neonatal demise of LPLO mice; these mice die within 24 hours of birth. All pups with the hLpLHBM/LpLO genotype survived and appeared normal. Postheparin plasma from hLpL ${ }^{\mathrm{HBM}} / \mathrm{LpL} 0$ mice contained $16.1 \pm 3.1 \mu \mathrm{mol} \mathrm{FFA} / \mathrm{ml} / \mathrm{h}$ of hLpL activity. This is comparable to the hLpL activity found in mice expressing nonmutated hLpL using the MCK promoter (ref. 20, $23.8 \mu \mathrm{mol} \mathrm{FFA} / \mathrm{ml} / \mathrm{h}$ ).

Interaction of $h L P L^{H B M}$ and $h L p L$ with lipoproteins in postheparin plasma of mice on the LPLO background. As shown in
Figure $2 \mathrm{c}\left(\mathrm{hLpL} \mathrm{LBM}^{\mathrm{HBM}} \mathrm{LpLO}\right)$ and Figure $2 \mathrm{~d}(\mathrm{hLpL} / \mathrm{LpLO})$, LPL activity coeluted with cholesterol-rich lipoproteins in both genotypes. In contrast to LpL in human postheparin plasma (35), the majority of LpL activity was associated with HDL, presumably because of the lack of sufficient amounts of apoB-containing lipoproteins to bind all the LPL. hLpL mass in both genotypes also coeluted with LDL and HDL; in the hLpL ${ }^{\mathrm{HBM}}$ there was no significant non-lipoprotein-associated $\mathrm{LPL}$ protein (data not shown). Thus, the carboxyl-terminal mutation in hLpL ${ }^{\mathrm{HBM}}$ did not significantly alter the enzyme's ability to bind to lipoproteins.

Northern blot. Northern blot analysis was performed to compare expression levels of the transgenes in hLpLHBM and hLpL mice. In skeletal muscle (Figure 3, lanes 1 and 2, 90-minute exposure) and heart (lanes 3 and 4, 16-hour exposure), more LpL mRNA was found in tissues from the hLpL ${ }^{\mathrm{HBM}}$ expressing mice (lanes 2 and 4 ). Neither transgene was expressed in kidneys (lanes 5 and 6, 16-hour exposure) or lungs (lanes 7 and 8).

Plasma lipids. On the wild-type background hLpL HBM did not significantly alter plasma TG or cholesterol levels (TG levels were $50 \pm 5$ in hLpL ${ }^{\mathrm{HBM}} / \mathrm{LpL} 2$ females and $51 \pm 5$ in LpL2 female littermates, $n=4$ each; TG was $65 \pm 17 \mathrm{in} \mathrm{hLpL} \mathrm{HBM}^{\mathrm{HB}} / \mathrm{LpL} 2$ males, $n=4$, and $81 \pm 17$ in LPL2 male littermates, $n=3$ ). However, on the heterozygous LpL knockout background hLpL ${ }^{\mathrm{HBM}}$ significantly reduced plasma TG levels (Table 1). In addition, plasma lipid values obtained from LpL1 littermates without the transgenes are shown; note that the littermates derived from breeding of $h L p L$ and $h L L^{H B M}$ had no significant differences in lipids. This suggests that there were not underlying genetic differences in the two lines of mice. On the homozygous LpL knockout background hLpL HBM mice were compared with hLpL transgenic mice and wild-type littermates. Both male and female hLpL ${ }^{\mathrm{HBM}} / \mathrm{LpL0}$ mice had higher levels of plasma 


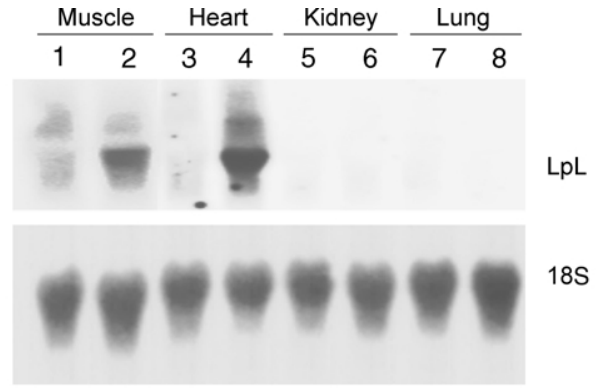

\section{Figure 3}

LpL RNA tissue levels of $h L p L^{H B M}$ and $h L p L$ transgenic mice on the LpL knockout background. Total RNA of the indicated tissues of $\mathrm{hLpL} \mathrm{HBM}^{\mathrm{L}} / \mathrm{LpL} 0$ and $\mathrm{hLpL} / \mathrm{LpL} 0$ mice was separated on a $1 \%$ agarose gel, blotted to a nylon membrane, and hybridized with the $\sim 500 \mathrm{bp}$ Rsrll/HindIII fragment of the hLpL minigene. Lanes 1 and 2 show a 90-minute autoradiography of the blot while the lanes 3-8 show a 16-hour exposure. hLpLHBM RNA is in lanes 2, 4, 6, and 8; hLpL-RNA is in lanes 1, 3, 5, and 7. For control, 18S RNA was hybridized with a complementary oligonucleotide.

TG than either hLpL/LpL0 or wild-type mice. This was due to an approximately twofold increase in VLDL TG $(P<0.001$ compared with hLpL/LpL0). Plasma cholesterol was similar in the two types of mice. In both female and male hLpL ${ }^{\mathrm{HBM}} / \mathrm{LpL0}$ mice VLDL cholesterol was significantly higher than in hLpL/LpLO $(P<0.03)$.

$L P L$ stability. The observation that there was a large amount of inactive LpL protein in preheparin plasma of mice expressing the hLpL ${ }^{\mathrm{HBM}}$ transgene suggested that this enzyme was less stable than hLpL. To test this, the stability of hLpL $\mathrm{HBM}^{\mathrm{HBM}}$ activity was analyzed in three ways. Figure $4 \mathrm{a}$ shows that medium from $\mathrm{hLpL}^{\mathrm{HBM}}$-expressing $\mathrm{CHO}$ cells lost lipolytic activity quicker than that from hLpL-expressing cells. Heparin stabilized both
hLpL and $h L p L^{\mathrm{HBM}}$, but the mutated LpL still lost activity more quickly (Figure $4 \mathrm{~b}$ ). When residual LpL activity produced by transfected cells was assayed after a 4-hour incubation at $4^{\circ} \mathrm{C}$, more than $80 \%$ of hLpL remained while less than $15 \%$ of the $\mathrm{hLpL}^{\mathrm{HBM}}$ activity was recovered (Figure 4c). Finally, we compared the LpLstability in postheparin plasma of mice expressing either nonmutated hLpL or hLpL ${ }^{\mathrm{HBM}}$ on the LpL0 background (Figure 4d). After 30 minutes of incubation at $37^{\circ} \mathrm{C}$, hLpL $\mathrm{LBM}^{\mathrm{HBM}}$ postheparin plasma contained an undetectable amount of activity, whereas postheparin plasma containing hLpL retained more than $70 \%$ of the initial LpL activity. Therefore in addition to decreasing LpL binding to heparin, mutation of the three basic amino acids in the carboxyl-terminus of $\mathrm{LPL}$ leads to a marked decrease of the enzyme's stability.

Release of $h L p L$ and $h L P L^{H B M}$ from muscle. To determine if the mutation in heparin binding also affects LPL association with muscle, the amount of mutated and hLpL dissociated from the muscle in $4^{\circ} \mathrm{C}$ media was assessed. Total immunoreactive $\mathrm{LPL}$ protein was greater in the $\mathrm{hLpL}^{\mathrm{HBM}}$ expressing muscle $(2.3 \pm 1.0$ versus $1.0 \pm 0.3 \mu \mathrm{g} / 100 \mathrm{mg}$ of tissue, $P<0.05, n=4$ for each group). More hLpL ${ }^{\mathrm{HBM}}$ protein dissociated into the medium during the incubation $(0.9$ versus $0.3 \mu \mathrm{g} / 100$ mg of muscle), and this was a greater percent of total LPL protein than was found in the hLpL-expressing tissues. Thus, $\mathrm{hLPL}^{\mathrm{HBM}}$ readily dissociated from binding sites within the muscle, and this was responsible for much of the hLpL ${ }^{\mathrm{HBM}}$ protein in preheparin blood.

Effects of $h L p L^{H B M}$ expression on postprandial lipemia. The role of heparin binding in the physiological targeting of lipoprotein lipids was tested in these mice. Female mice had similar plasma levels of FFAs after overnight fasting $\left(1.35 \pm 0.3 \mathrm{mmol} \mathrm{FFA} / \mathrm{l}\right.$ in $\mathrm{hLpL}^{\mathrm{HBM}} / \mathrm{LpLO}$,

\section{Table 1}

Plasma lipids on the LpL knockout background

TGs

\begin{tabular}{|c|c|c|c|c|c|c|c|c|c|c|}
\hline \multicolumn{2}{|c|}{ Background } & sex & $n$ & $h L p L^{H B M}$ & $n$ & littermates & $n$ & hLpL & $n$ & littermates \\
\hline \multirow[t]{2}{*}{ LpL1 } & Plasma & $f$ & 6 & $57.5 \pm 8.8^{A}$ & 6 & $106 \pm 18$ & 9 & $60.2 \pm 14.7^{A}$ & 5 & $105.8 \pm 9.7$ \\
\hline & & $\mathrm{m}$ & 8 & $87.6 \pm 15.2^{\mathrm{A}, \mathrm{B}}$ & 4 & $128.2 \pm 12.8$ & 8 & $53.6 \pm 5.3^{\mathrm{A}}$ & 6 & $125.3 \pm 29.3$ \\
\hline \multirow[t]{8}{*}{ LpL0 } & Plasma & $\mathrm{f}$ & 18 & $91.4 \pm 19.5^{C}$ & & & 10 & $54.3 \pm 9.8$ & & \\
\hline & & $\mathrm{m}$ & 15 & $102.9 \pm 14.7^{c}$ & & & 14 & $61.5 \pm 18.6$ & & \\
\hline & VLDL & $f$ & 18 & $55.3 \pm 11.4 \mathrm{C}$ & & & 10 & $24.6 \pm 5.8$ & & \\
\hline & & $\mathrm{m}$ & 15 & $73.9 \pm 14.2^{C}$ & & & 14 & $34.4 \pm 14.9$ & & \\
\hline & LDL & $f$ & 18 & $7.8 \pm 2.2$ & & & 10 & $8.0 \pm 1.3$ & & \\
\hline & & $\mathrm{m}$ & 15 & $10.3 \pm 3.9$ & & & 14 & $10.5 \pm 6.4$ & & \\
\hline & HDL & $\mathrm{f}$ & 18 & $1.6 \pm 1.5$ & & & 10 & $3.0 \pm 2.3$ & & \\
\hline & & $\mathrm{m}$ & 15 & $3.1 \pm 6.5$ & & & 14 & $3.3 \pm 7.2$ & & \\
\hline
\end{tabular}

Cholesterol

$\begin{array}{cccc}\text { LpL1 } & \text { Plasma } & f & 6 \\ \text { LpL0 } & \text { Plasma } & \mathrm{m} & 8 \\ & & \mathrm{~m} & 18 \\ & \text { VLDL } & \mathrm{f} & 18 \\ & & \mathrm{~m} & 15 \\ & \text { LDL } & \mathrm{f} & 18 \\ & \mathrm{~m} & \mathrm{~m} & 15 \\ & \mathrm{H} \text { L } & \mathrm{f} & 18 \\ & & \mathrm{~m} & 15\end{array}$

$\begin{array}{rlrl}71.4 & \pm 11.0 & 5 & 83.9 \pm 19.3 \\ 68.7 & \pm 14.5 & 6 & 83.7 \pm 20.9 \\ 68.1 & \pm 19.2 & & \\ 75.5 & \pm 15.8 & & \\ 10.5 & \pm 2.0 & & \\ 5.0 & \pm 3.1 & & \\ 17.2 & \pm 3.9 & & \\ 12.0 & \pm 3.7 & & \\ 42.0 & \pm 15.3 & & \\ 48.1 & \pm 10.6 & & \end{array}$




\section{Figure 4}

$\mathrm{LpL}$ stability. (a) Media from $\mathrm{CHO}$ cells expressing nonmutated $\mathrm{hLpL}$ (filled squares) and hLpL ${ }^{H B M}$ (open circles) were assayed for $L p L$ activity before and at the indicated time points during incubation at $37^{\circ} \mathrm{C}$. The activity before the incubation is set as $100 \%$. (b) $5 \mathrm{U}$ heparin $/ \mathrm{ml}$ were added to the cell culture media, then the experiment was performed as in a. (c) The stability of hLpL ${ }^{\mathrm{HBM}}$ (open bars) associated with the cell surface of $\mathrm{CHO}$ cells was compared with hLpL (filled bars). The initial cell-associated LpL activity was determined by incubating the cells with $20 \mathrm{U} / \mathrm{ml}$ of heparin for 30 minutes at $4^{\circ} \mathrm{C}$. This was set as $100 \%$. Then LpL activity in heparin-free media after a 4-hour incubation at $4^{\circ} \mathrm{C}$ with the cells is shown. The third bar indicates the activity released from the cell surface with heparin after the 4-hour incubation described before. (d) Stability of postheparin plasma from $\mathrm{hLpL} \mathrm{HBM}^{\mathrm{HB}} / \mathrm{LpL} 0$ was compared with $\mathrm{hLpL} / \mathrm{LpL0}$. The samples were assayed for $\mathrm{LpL}$ activity before incubation at $37^{\circ} \mathrm{C}$ and at the indicated time points. The initial activity was set to $100 \%$.

$n=5$, and $1.26 \pm 0.1 \mathrm{mmol} \mathrm{FFA} / \mathrm{l}$ in hLpL/LpL0, $n=4)$. These fatty acids are mainly derived from adipose tissue stores and not circulating lipoproteins. In contrast, significant differences between these two genotypes were found in the same mice in the postprandial state at 1 and 2 hours (Figure 5). The postprandial TG was also significantly different; 1 hour after gavage TG was 2.2 -fold higher in $\mathrm{hLpL}^{\mathrm{HBM}} / \mathrm{LpLO}(P<0.02)$, at the 2 -hour time point the elevation was 4.2 -fold $(P<0.02)$ and 3 hours postprandial the TG was still 1.4-fold higher $(P<0.02)$ in these mice. Fasting as well as postprandial FFA did not differ in LPL1 littermates of both types of mice ( $n=5$ each, data not shown). Therefore, although hLpL-expressing mice removed TG more efficiently, suggesting more active lipolysis, $\mathrm{hLpL}^{\mathrm{HBM}}$ mice had more fatty acids in their bloodstream. One explanation for these results is that more chylomicron lipolysis occurred away from the microvascular bed in muscle of hLpL ${ }^{\mathrm{HBM}} / \mathrm{LpL0}$ mice. This might have led to less uptake of the newly generated FFAs by muscle tissue.

Metabolism of intravenously injected rat chylomicrons, Intralipid and palmitate. Rat chylomicrons with $\left({ }^{3} \mathrm{H}\right)$ fatty acid labeled TG and $\left({ }^{14} \mathrm{C}\right)$ cholesterol were used to assess plasma lipoprotein turnover, generation of FFAs within the circulation, and organ lipid uptake. As shown in Figure 6a, the fractional catabolic rate of these particles $\left({ }^{3} \mathrm{H}\right.$-label) in $\mathrm{hLpL} \mathrm{HBM}^{\mathrm{HBp}} \mathrm{Lp} 0$ was significantly slower than that of hLpL/LpL0 mice $(0.14 \pm 0.02$ vs. $0.19 \pm 0.01$ pools $/ \mathrm{min}, P<0.01)$. However, significantly more fatty acids were generated in hLpL ${ }^{\mathrm{HBM}} / \mathrm{LpLO}(4,466 \pm 1,275$ $\mathrm{dpm} / \mathrm{ml}$ plasma vs. $1,180 \pm 1,278 \mathrm{dpm} / \mathrm{ml}$ plasma in hLpL/LpL0, $P<0.01$, Figure $6 \mathrm{~b}$ ). More of each label was found in muscles of hLpL/LpLO and less in the kidneys (Figure 6c). This suggested that hLpL increased uptake of both newly generated fatty acids and core lipids. This was confirmed by assessing the uptake of Intralipid. Over $99 \%$ of the label was cleared after 10 minutes after an intravenous injection in the two groups of mice. In skeletal muscle there was more than 1.5 -fold more fatty acid and cholesteryl ether uptake in hLpL/LpL0 compared with hLpL ${ }^{\mathrm{HBM}} / \mathrm{LpL0}$ mice $(P \leq 0.02)$. Interesting-
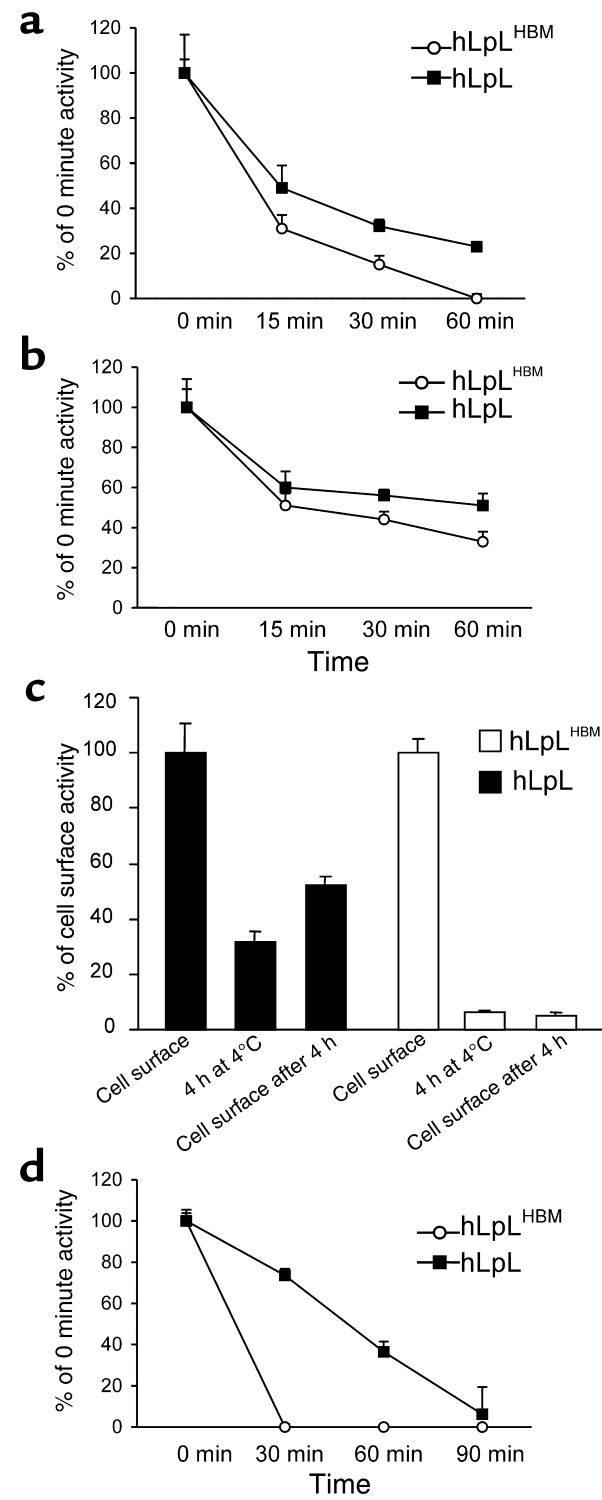

ly, kidneys of hLpL HBM /LpL0 mice accumulated 35\% more fatty acids $(P=0.05)$ and $50 \%$ more cholesteryl ether $(P<0.02)$ than kidneys of hLpL/LpL0 mice. This may have resulted from a greater amount of hLpL in kidneys from hLpL ${ }^{\mathrm{HBM}} / \mathrm{LpLO}$ mice $(377 \pm 42$ versus $221 \pm 34 \mathrm{ng} / 100 \mathrm{mg}$ tissue, $n=4, P=0.001)$. Therefore, the expression of the hLpL ${ }^{\mathrm{HBM}}$ transgene on the LPLO background leads to more FFAs in plasma, less lipid uptake in skeletal muscle, and more uptake in kidneys than overexpression of nonmutated hLpL.

As shown in Figure 6d, the plasma clearance of $\left({ }^{3} \mathrm{H}\right)$ palmitate was similar in the two types of mice. By 15 seconds after injection approximately $75 \%$ of the injected dose was cleared from the circulation $(74.3 \pm 5.9 \%$ in $\mathrm{hLpL} \mathrm{HBM}^{\mathrm{HB}} \mathrm{LpL0}$ and $75.6 \pm 6.6 \%$ in hLpL/LpL0). Two or three minutes after injection approximately $98 \%$ of the injected dose had been removed from the bloodstream of mice with both genotypes. Therefore, differences seen in postprandial FFA and in tissue lipid uptake from Intralipid emulsion in these mice were not due to dif- 


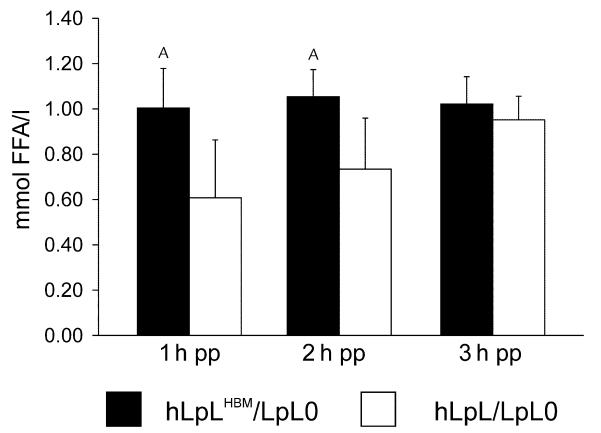

Figure 5

Postprandial FFAs. After 8 hours of daytime fasting, a gavage with $100 \mu \mathrm{l}$ corn oil was performed. Blood was withdrawn from the retroorbital plexus and plasma was assayed for FFA at the indicated time points. ${ }^{A} P<0.03$. $P p$, postprandial.

ferences in the mice's capability to remove fatty acids from the circulation.

Muscle histology. A final method to assess lipid metabolism of muscle was employed. Previous studies had shown that expression of hLpL on the wild-type background at levels similar to those of the hLpL ${ }^{\mathrm{HBM}}$ transgenic mice led to fatty acid-induced myopathic changes (20). Neither light microscopic analysis of routinely stained paraffin sections (Figure 7) nor further histochemical analysis on fixed and frozen sections as well as ultrastructural investigations on hand of semiand ultrathin sections of Epon-embedded samples by electron microscopy revealed any evidence for a lipolysis-induced myopathy comparable to that with overexpression of hLpL.

\section{Discussion}

These studies were performed to define the regions of $\mathrm{LPL}$ required for its high affinity binding to HSPGs and to define the importance of HSPG binding in LPL metabolism and physiological actions. Several basic amino acids in LpL were mutated and both in vitro and in vivo studies were performed. Our experiments showed the following: (a) The carboxyl-terminal region was important for LpL binding to heparin, and cells, (b) $\mathrm{hLpL} \mathrm{LBM}^{\mathrm{HBM}}$ was found in the bloodstream in preheparin blood, suggesting that endothelial HSPG binding was defective in vivo, (c) abnormal heparin binding still allowed production of active $\mathrm{LpL}$, although that $\mathrm{LpL}$ had reduced stability, (d) hLpL $\mathrm{HBM}^{\mathrm{HBM}}$ transgene produced active $\mathrm{LPL}$ in vivo and prevented the neonatal demise of LpL knockout mice, (e) the hLpL ${ }^{\mathrm{HBM}}$ transgene led to greater amounts of postprandial FFAs, suggesting that more lipolysis was occurring in the bloodstream rather that along the lumen of capillary endothelial cells, and (f) compared with mice expressing nonmutated hLpL,

\section{a}

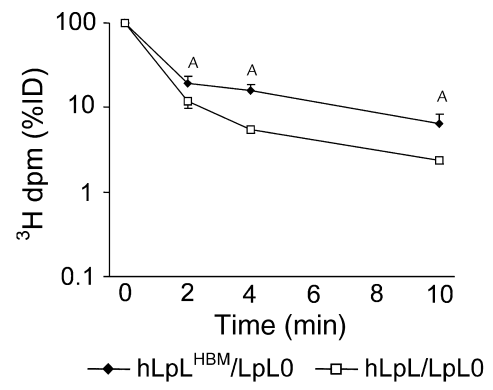

b

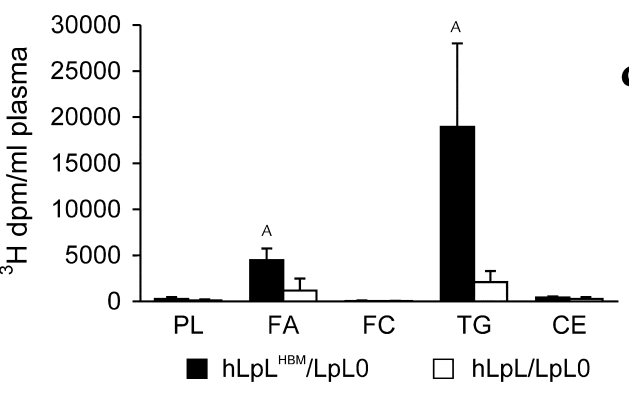

c

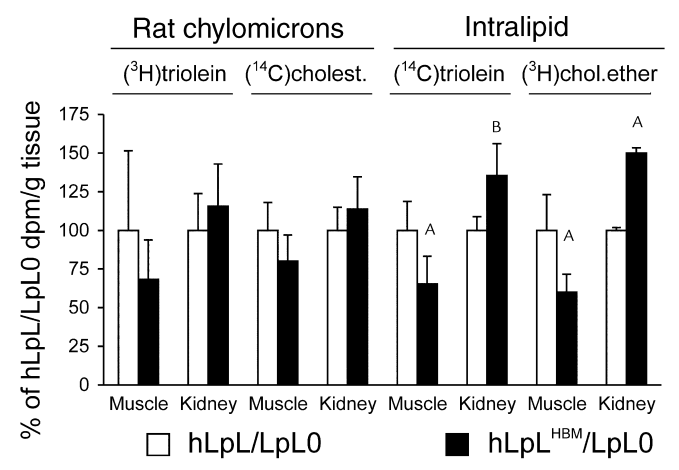

d

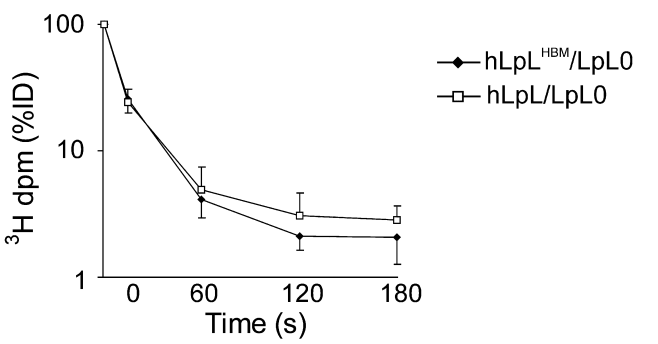

Figure 6

Rat chylomicron, Intralipid, and palmitate turnover studies. (a) Rat chylomicrons were labeled in vivo with $\left({ }^{3} \mathrm{H}\right)$ triolein, collected as described in Methods, and injected into the tail vein of hLpL $\mathrm{HBM} / \mathrm{LpLO}(n=5)$ and $\mathrm{hLpL} / \mathrm{LpL} 0(n=5)$ male mice. Blood was collected 2, 4 , and $10 \mathrm{~min}-$ utes after injection. Total plasma volume was calculated as $2.75 \%$ of body weight. Data are expressed as percentage of injected dose (ID). (b) A $100 \mu \mathrm{l}$ aliquot of the plasma from the 10-minute time point was lipid extracted and separated by TLC. The bars show the ${ }^{3} \mathrm{H} d p m$ per $\mathrm{ml}$ plasma for the indicated lipid. (PL, phospholipids; FA, fatty acids; FC, free cholesterol; CE, cholesterol esters). (c) Ten minutes after injection of labeled rat chylomicrons or Intralipid (injected into four $\mathrm{hLpL} H B M / L p L 0$ and four hLpL/LpL0 female mice), the mice were perfused with PBS and the indicated organs were taken out. Lipids were extracted with chloroform-methanol 2:1 (42) and counted. The data are expressed as percentage of hLpL/LpL0 dpm/g tissue. (d) $\left({ }^{3} \mathrm{H}\right)$ palmitate was complexed to fatty acid-free BSA and injected into four hLpL ${ }^{\mathrm{HBM}} / \mathrm{LpLO}$ and four $\mathrm{hLpL/LPLO}$ male mice. Mice were bled at the indicated time points. Data are expressed as percentage of injected dose. ${ }^{A} P \leq 0.02$; в $P \leq 0.05$. Cholest., cholesterol; chol.ether, cholesterol ether. 

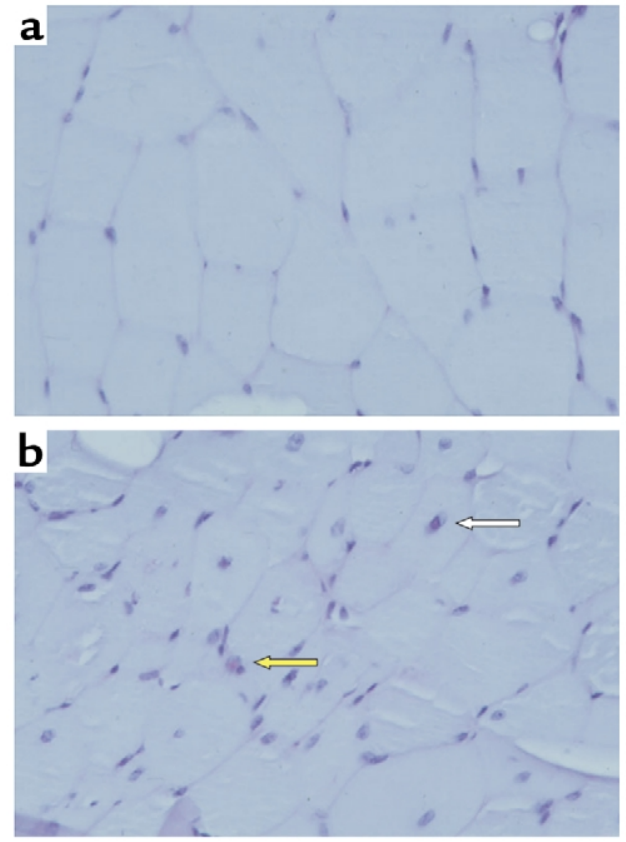

\begin{abstract}
Figure 7
Histological examination of muscle from $h L_{p L}{ }^{H M B}$ transgenic mice. Periodic-acid Schiff-stained (PAS-stained) sections of quadriceps muscles of an 8-month-old female hLpL HBM/LpL2 mouse. In most areas completely normal histology was found (a). Only in small areas of hLpL ${ }^{\mathrm{HBM}} / \mathrm{LpL} 2$ quadriceps some discrete, nonspecific myopathic alterations including more frequently centralized nuclei, a low number of in part atrophic muscle fibers, and some lipocytes in the endomysium could be found. Rarely a slight increase in glycogen storage could be detected (b). Compared with mice expressing high levels of $h L p L$, these findings are minimal. White arrow shows centralized nucleus, yellow arrow shows glycogen.
\end{abstract}

muscle expression of hLpL ${ }^{\mathrm{HBM}}$ led to less uptake of plasma lipids into muscle and, surprisingly, more lipid uptake in the kidney.

Our first objective was to determine whether the carboxyl region of hLpL is important for HSPG binding in vitro and in vivo. Cells expressing the $\mathrm{hLpL}^{\mathrm{HBM}}$ produced active enzyme protein, but the association of that protein with heparin affinity gel was markedly impaired. Inactive hLpL ${ }^{\mathrm{HBM}}$ protein in the preheparin plasma of transgenic mice eluted from the gel at about $0.4 \mathrm{M} \mathrm{NaCl}$. A second protein peak found in postheparin plasma eluted at $0.8-1 \mathrm{M} \mathrm{NaCl}$. This presumably dimeric LpL was clearly defective in heparin binding compared with normal LpL that eluted at greater than $1.2 \mathrm{M} \mathrm{NaCl}$. Thus, the carboxyl-terminal mutations led to production of a $\mathrm{LpL}$ molecule that was active and had normal interaction with plasma lipoproteins but was defective in heparin binding. Lookene et al. (19) investigated LPL heparin and HSPG binding properties using a Biacore system (Biacore AB, Uppsala, Sweden). They introduced mutations similar to ours (amino acids 403,405 , and 407 were changed to alanine) in an isolated $\mathrm{COOH}$-terminal $\mathrm{LpL}$ domain (amino acids 313-448). Although this mutated LpL fragment had virtually no heparin binding, they concluded that the contribution of the $\mathrm{COOH}$-terminal domain to heparin binding of $\mathrm{LpL}$ is about $20-40 \%$. We show that in vivo the mutation of the $\mathrm{COOH}$-terminal heparinbinding region markedly reduces heparin binding with several significant alterations in LpL's physiologic actions. However, our mutations did not completely eliminate the enzyme's binding to HSPGs.

The observation that a large amount of $\mathrm{hLpL}^{\mathrm{HBM}}$ was in the preheparin plasma of the transgenic mice was most consistent with reduced ability of this protein to remain associated with its capillary endothelial HSPG$\mathrm{LpL}$ binding site. This would allow the protein to enter the bloodstream without the usual infusion of heparin. Other studies confirmed that hLPL ${ }^{\mathrm{HBM}}$ dissociated from isolated skeletal muscle more readily. This undoubtedly led to some of the increased $\mathrm{LPL}$ protein found in preheparin plasma. At this time, we cannot definitely rule out that some of the preheparin $\mathrm{hLpL}{ }^{\mathrm{HBM}}$ was also due to an accompanying defect in removal of circulating LpL from the bloodstream. It may well be that binding to HSPGs in the sinusoids of the liver is a prerequisite for uptake and degradation of LpL by hepatocytes, since heparin infusion will block LPL removal from the bloodstream (12).

LpL, like a number of other proteins including growth factors and clotting factors, has a high affinity binding to heparin. There may be a number of physiologic reasons for this, and our data suggest that LpL heparin binding modulates its enzymatic activity and localizes the enzyme to tissues that are most important for uptake of lipoprotein-derived fatty acids. Concomitant with defective HSPG binding was a reduction in the stability of LPL activity; this was likely to have resulted from conversion of dimeric LpL to monomers. Thus, a fundamental role of HSPG binding illustrated in this study is maintenance of protein quaternary structure.

The physiological repercussions of reduced LpL association with HSPGs were also studied. Although hLpLHBM contained sufficient enzymatic activity to "rescue" LpL knockout mice, these animals were more hypertriglyceridemic than wild-type mice and mice expressing nonmutated hLpL. This suggested that the hLpLHBM/LpLO mice had a mild defect in TG lipolysis. Catabolism of both injected rat chylomicrons and postprandial TG was defective. Despite reduced lipolysis, plasma FFAs were higher in $\mathrm{hLpL}^{\mathrm{HBM}} / \mathrm{LpL0}$ mice. Since the clearance of labeled palmitate from the bloodstream was not delayed in these mice, this observation is most consistent with hydrolysis of TG in the circulating blood rather than in close proximity to the vascular endothelium in capillary beds. This presumably occurred during the short period of time between the release of the active enzyme from the endothelial surface and its inactivation.

$\mathrm{hLpL} \mathrm{HBM}^{\mathrm{HB}} / \mathrm{LpLO}$ and hLpL/LpL0 mice were compared to determine the effects of defective HSPG binding on tissue lipid uptake. Both histological studies and assessment of lipid turnover using a lipid emulsion showed less uptake of TG in the muscle of mice with 
the defective enzyme. Of interest, $h L \mathrm{pL}^{\mathrm{HBM}}$ led to more uptake in the kidney that we hypothesize is due to the presence of more LpL protein in that organ.

Studies of the structure-function relationships of complex proteins have several limitations: (a) the mutated region could affect a number of processes in addition to the one being investigated, and (b) multiple regions may be required for several biochemical interactions. Mutations in the carboxyl-terminal domain have several effects on $\mathrm{LpL}$ in addition to altering its heparin affinity. One region required for $\mathrm{LPL}$ receptor-related protein (LRP) binding and presumably other members of this family of receptors includes amino acids 404-430 (36), a region that includes the carboxyl terminal heparin-binding domain. It is, however, unlikely that defective LpL binding to LRP would alter LpL dissociation from heparin or muscle. Moreover, LPL-LRP interaction is unlikely to explain why the $h L \mathrm{pL}^{\mathrm{HBM}}$ leads to more fatty acids in plasma after an intravenous or oral fat load. A number of other mutations in LpL clearly affect its ability to bind to heparin (13-15). Thus, high affinity heparin binding is likely to require a number of LpL-heparin interactions. Although these sites could also be studied in vivo, our overall objective was to define the physiological significance of the major heparin-binding domain of LPL.

Understanding LpL-proteoglycan interactions may explain the regulation of $\mathrm{LPL}$ under physiologic and pathophysiologic conditions. LpL regulation is complex and much of the variation of LpL activity occurs at a posttranslational level. Decreases in LpL activity in adipose tissue during fasting $(37,38)$ and with diabetes (39) are not associated with reductions in $\mathrm{LpL}$ immunoreactive protein. Thus, it is likely that the dimeric LpL protein becomes inactivated; our data suggest that dissociation from proteoglycan binding sites on the endothelium, adipocytes, or interstitial matrix is likely to be responsible for this protein inactivation. A second example of the importance of LpLproteoglycan interaction is seen in the unusual patients with autoimmune syndromes leading to antibodies to heparin $(40,41)$. When these antibodies bind to HSPGs they presumably block access of LpL to these sites; the clinical presentation is severe hyperchylomicronemia due to lack of LpL-mediated TG hydrolysis and lack of LpL activity in the postheparin blood. Presumably the inability to bind to cell surface proteoglycans leads to rapid inactivation of $\mathrm{LPL}$ and an LpL-deficient phenotype.

In summary, we provide evidence that the carboxylterminal region of $\mathrm{LpL}$ is important for its association with HSPGs. Although the mutated region has several basic amino acids, it does not contain a classical heparin-binding motif. The physiological importance of defective heparin binding was demonstrated in genetically modified mice that only express the mutated LpL. Heparin binding via this carboxyl-terminal region is required to maintain LpL activity and probably prevents the conversion of the enzyme into inactive monomeric subunits. In addition, we show that optimal LpL binding to heparin is required to localize LpL to proteoglycan-rich cell membranes, leading to efficient targeting of newly created fatty acids to tissues. These studies are the first to our knowledge to assess the role of heparin binding in in vivo physiology and provide an approach to assess the importance of protein-proteoglycan interactions in other systems. Such interactions are critical for a number of other proteins including growth factors.

\section{Acknowledgments}

This study was supported by grants HL45095, 62301, and 56984 (SCOR) from the National Heart Blood and Lung Institute to I.J. Goldberg and HL 14990 to A. Bensadoun. E.P. Lutz is the recipient of a research grant $(\mathrm{Ra}$ 914/1-1) from the Deutsche Forschungsgemeinschaft. Histological preparations were supported by the Förderprogramm an den Medizinischen Einrichtungen Bonn (BONFOR-grant 154/41).

1. Goldberg, I.J. 1996. Lipoprotein lipase and lipolysis: central roles in lipoprotein metabolism and atherogenesis. J. Lipid Res. 37:693-707.

2. Iverius, P.H., Lindahl, U., Egelrud, T., and Olivecrona, T. 1972. Effects of heparin on lipoprotein lipase from bovine milk. J. Biol. Chem. 247:6610-6616.

3. Shimada, K., Gill, P.J., Silbert, J.E., Douglas, W.H., and Fanburg, B.L. 1981. Involvement of cell surface heparin sulfate in the binding of lipoprotein lipase to cultured bovine endothelial cells. J. Clin. Invest. 68:995-1002.

4. Saxena, U., Klein, M.G., and Goldberg, I.J. 1991. Identification and characterization of the endothelial cell surface lipoprotein lipase receptor. $J$. Biol. Chem. 266:17516-17521.

5. Parthasarathy, N., et al. 1994. Oligosaccharide sequences of endothelial cell surface heparan sulfate proteoglycan with affinity for lipoprotein lipase. J. Biol. Chem. 269:22391-22396.

6. Lookene, A., Chevreuil, O., Ostergaard, P., and Olivecrona, G. 1996. Interaction of lipoprotein lipase with heparin fragments and with heparan sulfate: stoichiometry, stabilization, and kinetics. Biochemistry. 35:12155-12163.

7. Conrad, H.E. 1998. Heparin-binding proteins. Academic Press Inc. San Diego, California, USA. 527 pp.

8. Vlodavsky, I., Miao, H.Q., Medalion, B., Danagher, P., and Ron, D. 1996. Involvement of heparan sulfate and related molecules in sequestration and growth promoting activity of fibroblast growth factor. Cancer Metastasis Rev. 15:177-186.

9. Cardin, A.D., and Weintraub, H.J. 1989. Molecular modeling of proteinglycosaminoglycan interactions. Arteriosclerosis. 9:21-32.

10. Margalit, H., Fischer, N., and Ben-Sasson, S.A. 1993. Comparative analysis of structurally defined heparin binding sequences reveals a distinct spatial distribution of basic residues. J. Biol. Chem. 268:19228-19231.

11. Wong, P., et al. 1995. Analysis of putative heparin-binding domains of fibroblast growth factor-1. Using site-directed mutagenesis and peptide analogues. J. Biol. Chem. 270:25805-25811.

12. Wallinder, L., Peterson, J., Olivecrona, T., and Bengtsson-Olivecrona, G. 1984. Hepatic and extrahepatic uptake of intravenously injected lipoprotein lipase. Biochim. Biophys. Acta. 795:513-524.

13. Ma, Y., et al. 1994. Mutagenesis in four candidate heparin binding regions (residues 279-282, 291-304, 390-393, and 439-448) and identification of residues affecting heparin binding of human lipoprotein lipase. J. Lipid Res. 35:2049-2059.

14. Hata, A., et al. 1993. Binding of lipoprotein lipase to heparin. Identification of five critical residues in two distinct segments of the amino-terminal domain. J. Biol. Chem. 268:8447-8457.

15. Berryman, D.E., and Bensadoun, A. 1993. Site-directed mutagenesis of a putative heparin binding domain of avian lipoprotein lipase. J. Biol. Chem. 268:3272-3276.

16. Davis, R.C., et al. 1992. Chimeras of hepatic lipase and lipoprotein lipase. Domain localization of enzyme-specific properties. J. Biol. Chem. 267:21499-21504.

17. Hill, J.S., et al. 1998. Subdomain chimeras of hepatic lipase and lipoprotein lipase. Localization of heparin and cofactor binding. J. Biol. Chem. 273:30979-30984.

18. Sendak, R.A., and Bensadoun, A. 1998. Identification of a heparin-bind- 
ing domain in the distal carboxyl-terminal region of lipoprotein lipase by site-directed mutagenesis. J. Lipid Res. 39:1310-1315.

19. Lookene, A., Nielsen, M.S., Gliemann, J., and Olivecrona, G. 2000. Contribution of the carboxy-terminal domain of lipoprotein lipase to interaction with heparin and lipoproteins. Biochem. Biophys. Res. Commun. 271:15-21

20. Levak-Frank, S., et al. 1995. Muscle-specific overexpression of lipoprotein lipase causes a severe myopathy characterized by proliferation of mitochondria and peroxisomes in transgenic mice. J. Clin. Invest. 96:976-986.

21. Obunike, J.C., et al. 2000. The heparin-binding proteins apolipoprotein $\mathrm{E}$ and lipoprotein lipase enhance cellular proteoglycan production. Arterioscler. Thromb. Vasc. Biol. 20:111-118.

22. Walsh, A., Ito, Y., and Breslow, J.L. 1989. High levels of human apolipoprotein A-I in transgenic mice result in increased plasma levels of small high density lipoprotein (HDL) particles comparable to human HDL3. J. Biol. Chem. 264:6488-6494.

23. Weinstock, P.H., et al. 1995. Severe hypertriglyceridemia, reduced high density lipoprotein, and neonatal death in lipoprotein lipase knockout mice. Mild hypertriglyceridemia with impaired very low density lipoprotein clearance in heterozygotes. J. Clin. Invest. 96:2555-2568.

24. Levak-Frank, S., et al. 1997. Induced mutant mice expressing lipoprotein lipase exclusively in muscle have subnormal triglycerides yet reduced high density lipoprotein cholesterol levels in plasma. J. Biol. Chem. 272:17182-17190.

25. Peterson, J., Fujimoto, W.Y., and Brunzell, J.D. 1992. Human lipoprotein lipase: relationship of activity, heparin affinity, and conformation as studied with monoclonal antibodies. J. Lipid Res. 33:1165-1170.

26. Hocquette, J.F., Graulet, B., and Olivecrona, T. 1998. Lipoprotein lipase activity and mRNA levels in bovine tissues. Comp. Biochem. Physiol. B Biochem. Mol. Biol. 121:201-212.

27. Goldberg, I.J., Paterniti, J.R., Jr., France, D.S., Martinelli, G., and Cornicelli, J.A. 1986. Production and use of an inhibitory monoclonal antibody to human lipoprotein lipase. Biochim. Biophys. Acta. 878:168-176.

28. Kako, Y., et al. 1999. Streptozotocin-induced diabetes in human apolipoprotein B transgenic mice. Effects on lipoproteins and atherosclerosis. J. Lipid Res. 40:2185-2194.

29. Havel, R.J., Eder, H.A., and Bragdon, J.H. 1955. The distribution and chemical composition of ultracentrifugally separated lipoproteins in human serum. J. Clin. Invest. 34:1345-1353.

30. Russell, W.E. 1988. Transforming growth factor beta (TGF-beta) inhibits hepatocyte DNA synthesis independently of EGF binding and EGF receptor autophosphorylation. J. Cell Physiol. 135:253-261.

31. Mortimer, B.C., Beveridge, D.J., Martins, I.J., and Redgrave, T.G. 1995. Intracellular localization and metabolism of chylomicron remnants in the livers of low density lipoprotein receptor-deficient mice and apoEdeficient mice. Evidence for slow metabolism via an alternative apoEdependent pathway. J. Biol. Chem. 270:28767-28776

32. van Bennekum, A.M., et al. 1999. Lipoprotein lipase expression level influences tissue clearance of chylomicron retinyl ester. J. Lipid Res. 40:565-574.

33. Merkel, M., et al. 1998. Catalytically inactive lipoprotein lipase expression in muscle of transgenic mice increases very low density lipoprotein uptake: direct evidence that lipoprotein lipase bridging occurs in vivo. Proc. Natl. Acad. Sci. USA. 95:13841-13846.

34. Coburn, C.T., et al. 2000. Defective uptake and utilization of long chain fatty acids in muscle and adipose tissues of CD36 knockout mice. J. Biol. Chem. 275:32523-32529.

35. Goldberg, I.J., Kandel, J.J., Blum, C.B., and Ginsberg, H.N. 1986. Association of plasma lipoproteins with postheparin lipase activities. J. Clin. Invest. 78:1523-1528.

36. Nielsen, M.S., et al. 1997. Segments in the C-terminal folding domain of lipoprotein lipase important for binding to the low density lipoprotein receptor-related protein and to heparan sulfate proteoglycans. J. Biol. Chem. 272:5821-5827.

37. Bergo, M., Olivecrona, G., and Olivecrona, T. 1996. Forms of lipoprotein lipase in rat tissues: in adipose tissue the proportion of inactive lipase increases on fasting. Biochem. J. 313:893-898.

38. Doolittle, M.H., Ben-Zeev, O., Elovson, J., Martin, D., and Kirchgessner, T.G. 1990. The response of lipoprotein lipase to feeding and fasting. Evidence for posttranslational regulation. J. Biol. Chem. 265:4570-4577.

39. Tavangar, K., et al. 1992. Regulation of lipoprotein lipase in the diabetic rat. J. Clin. Invest. 90:1672-1678.

40. Beaumont, J.L., and Lemort, N. 1974. Anti-heparin immunoglobulins, a factor of thrombosis, hyperlipemia and atherosclerosis. Pathol. Biol. (Paris). 22:67-76.

41. Glueck, C.J., et al. 1969. Acquired type I hyperlipoproteinemia with systemic lupus erythematosus, dysglobulinemia and heparin resistance. Am. J. Med. 47:318-324.

42. Kurlandsky, S.B., Gamble, M.V., Ramakrishnan, R., and Blaner, W.S 1995. Plasma delivery of retinoic acid to tissues in the rat. J. Biol. Chem. 270:17850-17857. 